Alonso Roque, J.I., Lavega Burgués, P., Etxebeste Otegui, J. \& Lagardera Otero, F. (2020). La huella deportiva como factor emocional para la formación docente universitaria en Educación física. Revista Electrónica Interuniversitaria de Formación del Profesorado, 23(3), 169-186.

DOI: https://doi.org/10.6018/reifop.366371

\title{
La huella deportiva como factor emocional para la formación docente universitaria en Educación Física
}

\author{
José Ignacio Alonso Roque ${ }^{1}$, Pere Lavega Burgués², Joseba Etxebeste Otegui ${ }^{3}$, \\ Francisco Lagardera Otero ${ }^{4}$ \\ ${ }^{1}$ Universidad de Murcia, ${ }^{2}$ Instituto Nacional de Educación Física de Cataluña, \\ ${ }^{3}$ Universidad del País Vasco, ${ }^{4}$ Instituto Nacional de Educación Física de Lérida
}

\section{Resumen}

Los juegos deportivos tradicionales generan interacciones motrices entre los participantes que activan procesos cognitivos, sociales y afectivos de gran interés para conocer las relaciones humanas en la formación docente universitaria. Este trabajo estudia el impacto emocional que suscitan las interacciones motrices y el historial deportivo (huella deportiva) en alumnos universitarios en formación. Para ello se analizan las interacciones motrices cooperativas y de oposición y distintos tipos de historial deportivo en un marco lúdico. Participaron 241 alumnos y se analizó la intensidad emocional a través del cuestionario Games and Emotions Scale (GES). El análisis de los datos siguió un modelo de ecuaciones de estimación generalizadas (GEE) (familia Gausiana). Las vivencias positivas fueron mayoritarias en los dos tipos de interacciones motrices experimentadas, siendo la vivencia cooperativa la más intensa. La experiencia motriz previa modificó la vivencia de emociones. El conocimiento de las características emocionales en ambas interacciones motrices y también la huella deportiva permite aplicar a futuros programas formativos contenidos específicos para mejorar la gestión de las relaciones motrices.

\section{Palabras clave}

Huella deportiva; emociones; juegos deportivos tradicionales, educación física. 


\section{The sport track as an emotional factor for university teacher formation in physical education}

\section{Abstract}

Traditional sports games generate motor interactions between participants that activate cognitive, social and affective processes of great interest to understand human relationships in university teacher formation. This work studies the emotional impact of motor interactions and sports history (sport track) on university students in formation. To this end, the cooperative and opposition motor interactions and different types of sports history are analysed in a playful context. A total of 241 student's participated and emotional intensity was analysed using the Games and Emotions Scale (GES) questionnaire. The data analysis followed a model of generalized estimation equations (GEE) (Gaussian family). The positive experiences were majority in the two types of motor interactions experienced, being the cooperative experience the most intense. The previous physical event modified the experience of emotions. The knowledge of the emotional characteristics in both motor interactions and also the sports track allows to apply to future training programs specific contents to improve the management of motor relationships.

\section{Key words}

Sports track; emotion; traditional sports games; physical education.

\section{Introducción}

Cuando dos o más personas interactúan en un juego motor las relaciones motrices se acompañan al mismo tiempo de procesos biológicos, cognitivos, afectivos y sociales que dan testimonio de la naturaleza de esa relación humana (Alonso, Lavega y Gea, 2013; Bächler y Poblete, 2012). Para comprender dichos procesos asociados al diálogo interpersonal es preciso desvelar las propiedades del juego en el que se participa (juego), así como las características de los protagonistas (actores). Esta doble perspectiva corresponde a la noción de situación motriz que acuña la teoría de la acción motriz (Parlebas, 2001) donde "existe interacción motriz cuando el comportamiento motor de un individuo influye de manera observable en el de los demás participantes" (Parlebas, 2001, p. 269).

De los posibles contextos sociales en los que acontecen relacionales humanas, el juego deportivo tradicional (JDT) desencadena un repertorio extraordinario de maneras distintas de dialogar con los demás. Se trata de un legado cultural de manifestaciones motrices en las que las personas mientras juegan dan testimonio de rasgos culturales que caracterizan la manera de relacionarse de esa sociedad. De ahí que sea un recurso de primer orden en educación física para estudiar el torrente emocional que se desencadena entre el alumnado (Sáez de Ocáriz, Lavega, Costes y Serna, 2014; Jaqueira, Lavega, Lagardera, Araujo y Rodrigues, 2014).

No obstante, no abundan estudios que aborden las emociones ante los JDT, a excepción de investigaciones sobre regulación emocional en sesiones de educación física (Liukkonen, Barkoukis, Watt \& Jaakkola, 2010). Por este motivo el presente artículo profundiza en la doble perspectiva que contempla la noción de situación motriz. Por una parte, atendiendo a las características del sistema (juego) se pretende estudiar la relación existente entre dos clases distintas de interacción motriz; los JDT de oposición y los JDT cooperativos, 
practicados con o sin competición y la vivencia emocional de sus protagonistas. Paralelamente, considerando las características de los actores se pretende estudiar si la vivencia emocional es distinta en función del género y de la huella deportiva de esos sujetos.

\section{Cooperar u oponerse en los juegos deportivos tradicionales}

Un JDT es cooperativo si dos o más personas mantienen una interacción motriz positiva, basada en la ayuda mutua, destinada a superar un reto común, mediante acciones motrices de contacto corporal directo (e.g., sujetar a otra persona en una torre humana) o a través del intercambio de objetos (e.g., pasar un balón sin que caiga al suelo). Sería de oposición si dos o más personas interactúan motrizmente tratando de evitar que los rivales consigan su objetivo, ya sea a través de un enfrentamiento cuerpo a cuerpo (e.g., judo) o mediatizado por objetos (e.g., tenis).

La cooperación ha tenido una presencia tímida en el currículum oficial de educación física, considerada como una manifestación menor al compararse con otras modalidades, principalmente deportivas (Lavega et al., 2011). Por otra parte, los JDT de oposición se han visto como recursos poco apropiados por la existencia de un rival. Ambas clases de JDT pueden realizarse con presencia o ausencia de competición. Serán competitivos si los participantes orientan sus intervenciones hacia un resultado final que resume el éxito o fracaso en ese enfrentamiento. En cambio, la competición está ausente cuando las partidas carecen de un marcador final; sería el caso en el que los oponentes participan en secuencias cíclicas de juego con intercambios de roles (perseguidor y perseguido) sin que al final haya ganadores o perdedores (Lavega, Alonso, Etxebeste, Lagardera \& March, 2014).

La vivencia emocional en los juegos cooperativos y en los de oposición: Perspectiva de género

Tomar en consideración la perspectiva de género exige reconocer que una persona se comporta como hombre o mujer en función de la construcción social que ha ido tejiendo el entorno cultural en el que vive. Los hombres y las mujeres aprenden a comportarse como tales en función de unas expectativas, dando lugar a conductas propias de hombres (masculinidad) y de mujeres (feminidad). Desde esta perspectiva, la práctica de un determinado tipo de deportes o actividades lúdicas contribuye a que cada persona adquiera una construcción social de esa experiencia y también de las emociones que le acompañan (Harris, 1981).

La construcción social de la feminidad parece favorecer la práctica de aquellas actividades que enfatizan el equilibrio, la coordinación, la flexibilidad y la gracia, tales como la gimnasia, el patinaje sobre hielo o la natación sincronizada. Estos ejemplos constituyen deportes cooperativos, en los que las aptitudes mencionadas cuentan con mayor premio compitiendo. Por ejemplo, con participantes universitarios se encontraron tendencias hacia la cooperación teniendo en cuenta mayores intensidades emocionales positivas en juegos tradicionales cooperativos por parte de las chicas (Lavega, Araujo y Jaqueira, 2013; Lavega, et al., 2011; Mateu, Romero-Martín, Gelpí, Rovira y Lavega, 2014). También indican la predisposición masculina a jugar con adversarios a los que superar y a la existencia de un marcador. Estudios realizados en situaciones lúdicas en oposición (Alonso et al., 2013) mostraron que las intensidades emocionales positivas y negativas de los hombres fueron más elevadas que las de sus compañeras. Mientras las mujeres tienden a expresar las emociones, los hombres aprenden a excluirlas, lo que origina una desigualdad entre chicos y chicas (Fabes y Martin, 1991; Simon y Nath, 2004). 
Los hombres expresan emociones positivas con más frecuencia que las mujeres, quienes tienden a expresar más regularmente emociones negativas (Kelly y Hustson-Comeaux, 1999). Más recientemente, en cooperación, las chicas las viven de forma más positiva, ya que puntuaron alto en emociones como alegría o felicidad (Lavega et al., 2014). Estudios como los de Alonso et al. (2013), indicaron que las intensidades emocionales en determinados juegos de oposición se asociaron al género masculino en mayor medida. Etxebarria, Apodaca, Eceiza, Fuentes y Ortiz (2003), con niños y niñas de 7 a 9 años, señalan que las niñas puntuaron más altas en las escalas de tristeza y focalización de la atención. Así mismo, fueron evaluadas por sus maestros como más prosociales, menos agresivas con sus compañeros y más cooperativas con el profesorado.

Tampoco conviene obviar el efecto que sobre el aprendizaje tienen emociones que suscitan las experiencias deportivas ya que "Las emociones negativas (ansiedad, el sentido de inutilidad o la irritación) constituyen un potente factor de incomodidad en el aprendizaje de cualquier contenido porque atrapan la atención" (Velásquez y Guillén, 2007, p. 120). Se observan diferencias de género en diversas esferas de la vida, que luego no se ven refrendadas claramente en el contexto de una interacción motriz. Parecería que el género no es una variable determinante, solo orienta inicialmente a unas vivencias a las mujeres, más cooperativas.

La vivencia emocional en los juegos con presencia o ausencia de competición: Perspectiva de género

Cuando las mujeres y los hombres participan en situaciones competitivas donde se puede ganar o perder, la respuesta emocional suele ser desigual. Burton-Chellew, Gunnthorsdottir y Rapoport (2006), nos indican la mayor intensidad emocional en competición, por lo que la perspectiva de género, aportaría más luz sobre este aspecto. Ambos se socializan a través de la práctica en juegos tradicionales y deportes reproduciendo los valores y manifestando las emociones asociadas culturalmente a su género (Etxebeste, 2012). Ante una situación competitiva las mujeres son más contenidas al expresar emociones positivas en la victoria e interiorizan más las emociones negativas asociadas a perder, con una respuesta intensa pero dirigida hacia sí mismas. Los hombres reaccionan ante la victoria o la derrota de forma más extrovertida y ostensible. Al no competir el interés por ese tipo de prácticas es distinto en ambos géneros (Lavega, et al., 2014) y siempre los chicos han marcado puntuaciones más elevadas ante situaciones de ganar o perder los juegos sobre todo cuando eran con adversarios.

La vivencia emocional en los juegos deportivos tradicionales en función de la huella deportiva

Como en otras facetas vitales, nuestro pasado y experiencias determinan muchas decisiones y vivencias del presente. Planteamos la hipótesis de que en función de los antecedentes deportivos la persona puede orientar de manera distinta su vivencia motriz y por consiguiente su vivencia emocional y su formación.

Atendiendo a Lavega et al. (2014), se considera oportuno identificar dos criterios complementarios al examinar la incidencia de la huella deportiva en la vivencia emocional. Por una parte referido al tipo de interacción motriz deportiva en el que se ha especializado la persona; es decir, si se ha participado principalmente en un deporte que no exige interactuar motrizmente con los demás (e.g., carreras cortas de atletismo); o si ha intervenido en una de las tres opciones de los deportes sociomotores: deportes cooperativos (e.g., natación sincronizada); deportes de oposición (e.g., judo) o en deportes de cooperación-oposición (e.g., fútbol). En este sentido Del Pino, Gómez, Moreno y Gálvez (2009) examinan la inteligencia emocional entre deportistas con antecedentes baloncesto y 
el futbol, con otros que participan en deportes individuales (natación y concursos de atletismo). Se observa que los primeros obtienen puntuaciones más altas en inteligencia emocional que los que realizan deportes psicomotores.

Por otra parte, la huella deportiva puede estar asociada al nivel de experiencia acumulada en una modalidad deportiva concreta (Gutiérrez, González, García y Mitchell, 2011). Starkes y Ericsson (2003) plantean la necesidad de identificar y regular los estados emocionales en los deportistas expertos y novatos. Un experto, con una destacable huella deportiva, podría identificar sus emociones, así como regular y controlar mejor la excitación emocional durante el juego que un novato.

\section{Descripción general de los dos estudios}

Se han realizado dos estudios con el fin de conocer las características de los dos tipos de relaciones motrices abordadas. Se han diseñado dos investigaciones con el mismo procedimiento e instrumentos para analizar las vivencias emocionales respecto a las variables huella deportiva, tipo de relación motriz y género. Participaron tres grupos de estudiantes pertenecientes a cuatro universidades españolas localizadas en tres Comunidades Autónomas distintas (Cataluña, Región de Murcia y Andalucía).

Los objetivos generales fueron los siguientes:

1. Analizar el efecto de juegos deportivos tradicionales de cooperación y de oposición en la vivencia emocional.

2. Examinar la incidencia de la presencia o ausencia de competición en la vivencia emocional.

3. Analizar la influencia de la huella deportiva y el género en la vivencia de emociones en juegos deportivos tradicionales de oposición y en juegos deportivos cooperativos.

\section{Metodología}

En ambos estudios se siguió el mismo procedimiento de recogida de datos y análisis estadístico. Posteriormente se indicarán los participantes y resultados de cada uno de ellos.

Instrumentos y procedimiento. Estudio 1 y 2

Para la identificación de la intensidad emocional se empleó la escala GES (Lavega et al., 2013). Al finalizar cada juego, los alumnos marcaban el nivel de intensidad (de 0 a 10) que habían experimentado en cada una de las trece emociones (Bisquerra, 2003). El cero significaba que no se había sentido esa emoción, y el diez implicaba que se había vivido con máxima intensidad. Los JDT se realizaron durante cuatro sesiones de 1,5 horas. Los criterios de inclusión de los JDT fueron: (a) JDT en la cultura española sin sesgo de género; (b) debían representar al dominio de oposición (Estudio 1) y de cooperación (Estudio 2); (c) realizados en espacios deportivos estandarizados; (d) en la $1^{\text {a }}$ y $3^{\text {a }}$ sesión se emplearon juegos con competición; para la $2^{\mathrm{a}}$ y la $4^{\mathrm{a}}$ fueron juegos sin un ganador final. En los distintos grupos de alumnos participantes se siguió un orden diferente en los JDT, paliando el efecto de arrastre en los datos obtenidos. Las sesiones fueron dirigidas por el profesor habitual de cada grupo, el cual presentaba el juego explicando las reglas y solventando dudas. El profesor no intervenía motivando, ayudando, ni aportando feedback. 


\section{Análisis estadístico. Estudio 1 y 2.}

La normalidad de los datos se analizó a través de la prueba Kolmogorov-Smirnov, determinándose que los datos no seguían una distribución normal en ambos estudios. Se siguió un modelo basado en ecuaciones de estimación generalizadas (GEE) para tener en cuenta la correlación entre las puntuaciones del mismo sujeto y la distribución asimétrica. Se utilizaron distribuciones de la familia Gausiana, con una estructura de correlación intercambiable. Se aplicaron comparaciones múltiples post hoc en todos los factores de más de dos categorías. Se empleó el software estadístico SPSS v.19.0. El modelo consideró cinco factores o variables independientes. Dos factores intra-sujetos: 1) Tipo de resultado (ganar, perder y juego sin competición); y 2) Tipo de emoción (positiva, negativa y ambigua); y dos factores entre-sujetos: 1) Género (masculino/femenino); 2) Historial deportivo (tipo de antecedentes deportivos). La variable dependiente fue la intensidad emocional. Los resultados de las interacciones entre las variables fueron acompañados por el tamaño del efecto basados en la d de Cohen, adaptado por Wolf (1986) para medidas repetidas.

\section{Estudio 1. Juegos de oposición y vivencia emocional}

En el primer estudio se analizaron los datos correspondientes a los JDT de oposición, con y sin competición. Los JDT de oposición muestran presencia de adversario directo sobre el que las acciones motrices propias pasan por las de los adversarios. La presencia o ausencia de competición se determinó como la existencia o no de un marcador final que permitía clasificar a los jugadores en ganadores o perdedores del juego.

\section{Participantes y procedimiento. Estudio 1.}

El estudio se realizó con 169 alumnos (28 chicas y 141 chicos; $19-22$ años; Medad $=19.6$ años, DT $=2.33)$. El análisis estadístico de la intensidad emocional se realizó sobre un total de 42185 valoraciones. Todos los participantes fueron estudiantes de Grado en Educación Primaria-Educación física y de primer curso en Ciencias de la actividad física y del deporte de dos universidades españolas localizadas en la Región de Murcia y Andalucía. Se obtuvieron los consentimientos informados de participación voluntaria de los participantes y la investigación fue aprobada por el comité de ética de la Universidad de Murcia.

\section{Resultados. Estudio 1}

Los participantes (Tabla 1) mostraron diferencias significativas en intensidad emocional ( $p<$ .001). No se encontraron diferencias en función del género $(p<.936)$. Respecto a la huella deportiva, los alumnos en oposición mostraron diferencias significativas en función del deporte practicado ( $\mathrm{p}$. .001). Cuando se relacionaron las variables de huella deportiva, los tipos de emociones y el género registraron diferencias en la intensidad emocional respecto a esta variable.

La visión general del modelo permite analizar el comportamiento de las emociones ya que se observaron diferencias significativas en cuanto a intensidad emocional $(p<.01)$. 
Tabla 1.

Contrastes de los efectos del modelo en Oposición

\begin{tabular}{|l|r|r|r|}
\hline \multirow{2}{*}{ Origen } & \multicolumn{2}{|l|}{ Tipo III } & \\
\cline { 2 - 5 } & $\begin{array}{c}\text { Chi- } \\
\text { cuadrado } \\
\text { de Wald }\end{array}$ & gl & \multicolumn{1}{l|}{ Sig. } \\
\hline (Intersección) & 327.018 & 1 & $<.001$ \\
\hline Tipo de emoción & 105.480 & 2 & $<.001$ \\
\hline Género & 4.414 & 1 & .936 \\
\hline Tipo resultado & 2.660 & 2 & .264 \\
\hline Huella deportiva (Tipo deporte practicado) & 42.012 & 3 & $<.001$ \\
\hline Tipo de emoción *Huella dep.(Tipo deporte practicado) & 13.612 & 6 & .034 \\
\hline Género * Huella dep.(Tipo deporte practicado) & 7.378 & 2 & .025 \\
\hline Huella dep.(Tipo deporte practicado) * Tipo de resultado & 6.525 & 6 & .367 \\
\hline
\end{tabular}

Tipos de emociones suscitadas en los juegos deportivos de oposición

Los resultados muestran que las intensidades en emociones positivas fueron las más elevadas ( $p<.001$ ), seguidas por las emociones ambiguas y las negativas (Figura 1, $M=4.76$; DT $=.401, p<.001, d=4.46)$.

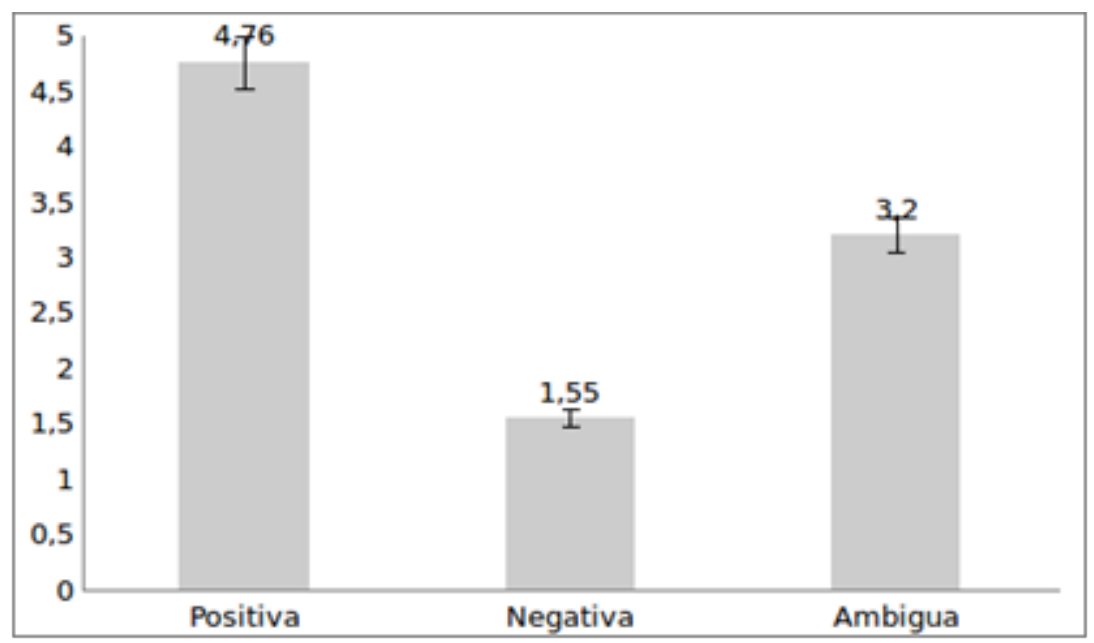

Figura 1. Intensidades de los tres tipos de emociones para los juegos de oposición.

\section{Emociones y competición}

Teniendo en cuenta la presencia o ausencia de la competición en el juego, se observó que las emociones fueron significativamente más intensas $(p<.001)$ al competir y ganar $(M=$ $3.27, \mathrm{DT}=.368, \mathrm{~d}=1.50)$ que al perder $(M=3.14, \mathrm{DT}=.380, \mathrm{~d}=.33)$. 


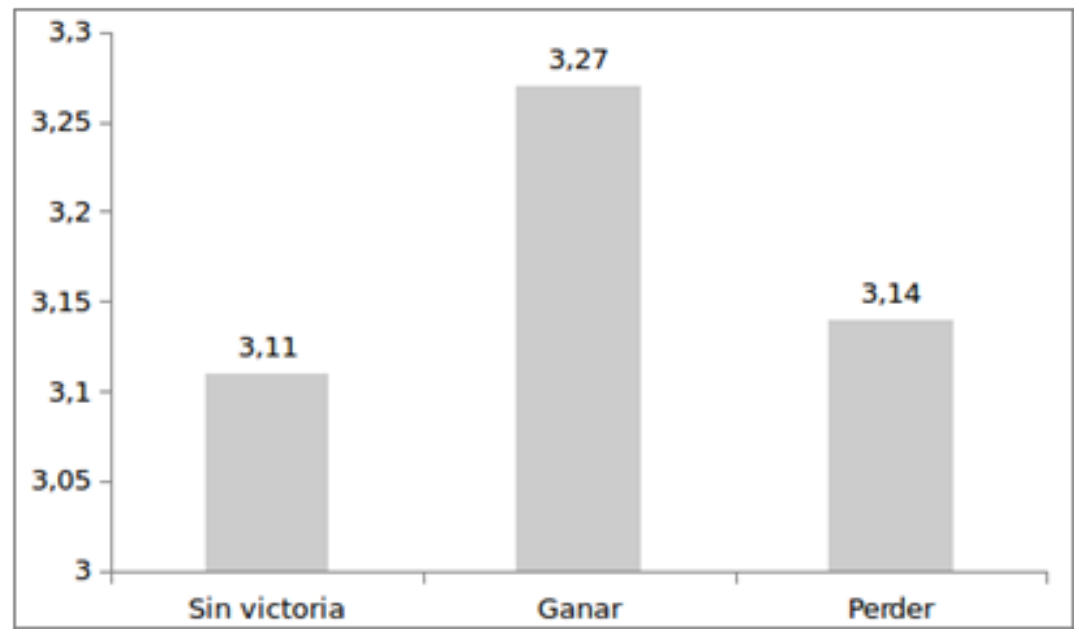

Figura 2. Intensidades emocionales en función del resultado del juego.

\section{Emociones y huella deportiva}

El hecho de contar con historial deportivo sugiere que el tipo de deporte practicado ha tenido influencia en la intensidad emocional. Los alumnos con un historial basado en deportes psicomotores puntuaron las emociones por debajo del resto de dominios $(M=2.4$; DT $=.223, p<.001, d=.47)$. Los participantes con historial en deportes cooperativos $(M=$ 3.48) no mostraron diferencias significativas en relación a los que tenían antecedentes en deportes psicomotores $(M=2.4, p=.214)$. Los participantes con historial en deportes de cooperación-oposición $(M=3.5, \mathrm{DT}=.326, \mathrm{p}<.001, \mathrm{~d}=4.44$.) sí mostraron diferencias significativas en relación a los que tenían antecedentes en deportes psicomotores. Se puede indicar que hay una tendencia a mostrar mayores intensidades emocionales cuando los historiales cuentan con el factor "oposición" respecto a los historiales sociomotores y psicomotores.

\section{Emociones y huella deportiva en función del género}

Según la interacción de la modalidad practicada, los chicos con un historial de deportes psicomotores obtuvieron menores intensidades emocionales $(M=2, D T=.224, p<.012, d=$ 2.59) con respecto a los mismos historiales femeninos. Del mismo modo, los historiales de oposición $(M=3.35$, $D T=.224, p<.001, d=2.28)$ y de cooperación con oposición $(M=3.68$, $\mathrm{DT}=.393, \mathrm{p}<.001, \mathrm{~d}=2.77)$ mostraron para los chicos mayores intensidades emocionales que para aquellos que mostraban una huella deportiva psicomotriz.

Los juegos de oposición fueron una experiencia agradable cuando actuaron contra un adversario. El modelo general también muestra que el tipo de resultado no fue una variable significativa para los alumnos. Dentro de las variables relacionadas con la huella deportiva existieron diferencias cuando se tuvo en cuenta el tipo de deporte practicado asociado al resultado final. De igual forma, el género solo determinó la vivencia si se tienen en cuenta los tipos de historial deportivo. Respecto a la existencia de marcador, ganar en un juego es el gran desencadenante emocional, siendo un resultado coherente con la cultura deportiva dominante. Perder o jugar sin marcador implicó una vivencia similar, lo que indica que jugar sin una comparación final resulta de interés, al desarrollar competencias, seguridades, 
autoconcepto, diversión y participación. El presente estudio indica que cuando aparece un adversario, o bien se comparan los resultados, esta tendencia se ve algo modificada.

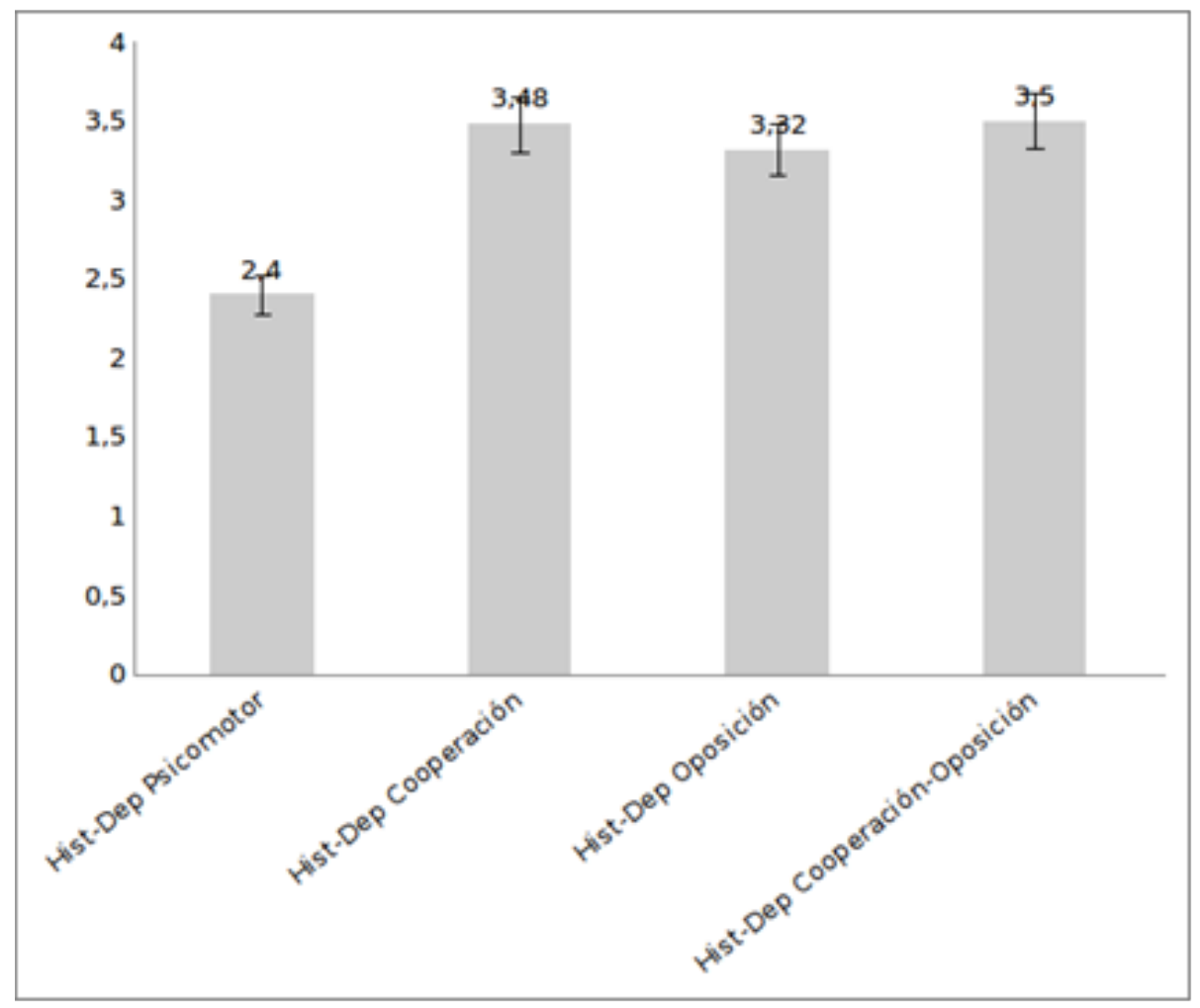

Figura 3. Valores en intensidad emocional el tipo de historial deportivo.

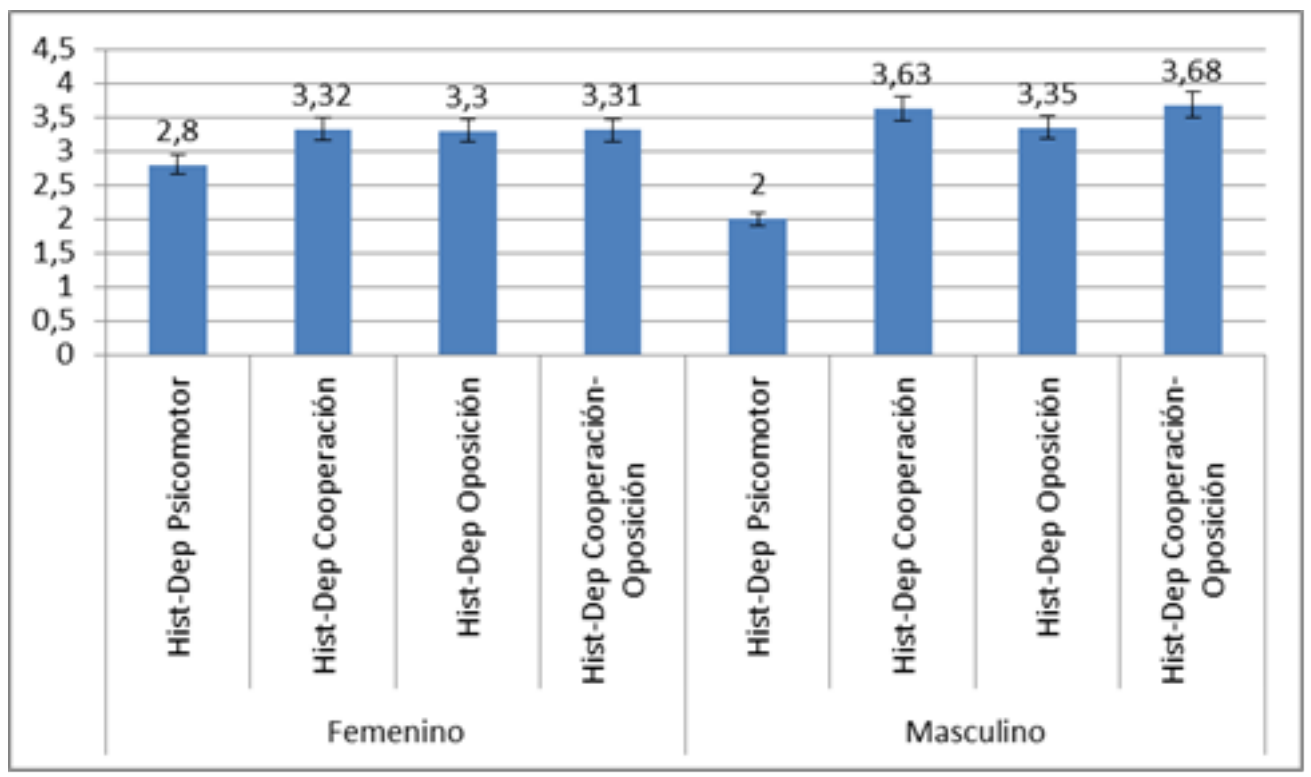

Figura 4. Intensidades emocionales para las interacciones motrices de oposición teniendo en cuenta el género y el tipo de historial deportivo. 
Al jugar en oposición existe una tendencia a mayores intensidades emocionales cuando los historiales deportivos cuentan con el propio factor de la oposición. Sin embargo, las personas con historiales deportivos cooperativos no muestran diferencia alguna respecto de los historiales con deportes psicomotores.

\section{Estudio 2. Juegos de cooperación y vivencia emocional}

En el segundo estudio se analizó la vivencia emocional ante la realización de juegos cooperativos. Los juegos deportivos cooperativos se caracterizan por la existencia de relaciones de colaboración sin oposición por parte de otros jugadores. No obstante, encontramos juegos cooperativos en los que pueden existir equipos que buscan una colaboración óptima para conseguir la victoria (gimnasia rítmica por equipos).

\section{Participantes Estudio 2}

El estudio se realizó con 70 alumnos (41 chicos y 29 chicas) de edades comprendidas entre 19-22 años (Medad = 19.6 años, DT = 2.33). El análisis estadístico de la intensidad emocional se realizó sobre un total de 21021 valoraciones. Todos los participantes fueron estudiantes de primer curso en Ciencias de la actividad física y del deporte de la Universidad de Lleida. Se obtuvieron los consentimientos informados de participación voluntaria de los participantes y la investigación fue aprobada por el comité de ética de la Universidad de Lleida.

\section{Resultados Estudio 2.}

Los resultados muestran que los alumnos que practicaron juegos de colaboración (Tabla 2) marcaron intensidades emocionales diferentes $(p<.001)$. Además, se encontraron diferencias en función del tipo de resultado (ganar, perder o jugar sin marcador) $(p<.001)$. Teniendo en cuenta las variables relacionadas con la huella deportiva previa, los alumnos en cooperación, mostraron diferencias significativas en función del deporte practicado $(p<$ .001) y huella deportiva-experiencia en años $(p<.021)$. Los tipos de emociones y el tipo de resultado, mostraron diferencias en la intensidad emocional cuando interaccionaron con la variable "Tipo de deporte practicado" ( $\mathrm{p}=.049$ y $\mathrm{p}=.002$, respectivamente).

Tabla 2.

Contrastes de los efectos del modelo para el dominio de Cooperación

\begin{tabular}{|c|c|c|c|}
\hline \multirow[b]{2}{*}{ Origen } & \multicolumn{3}{|l|}{ Tipo III } \\
\hline & $\begin{array}{l}\text { Chi-cuadrado } \\
\text { de Wald }\end{array}$ & gl & Sig. \\
\hline (Intersección) & 138.556 & 1 & $<.001$ \\
\hline Tipo de emoción & 109.985 & 2 & $<.001$ \\
\hline Género & .987 & 1 & .320 \\
\hline Tipo de resultado & 30.355 & 2 & $<.001$ \\
\hline Tipo deporte practicado & 25.136 & 3 & $<.001$ \\
\hline Huella Deportiva & 7.685 & & .021 \\
\hline
\end{tabular}


Tabla 2.

Contrastes de los efectos del modelo para el dominio de Cooperación

\begin{tabular}{|l|l|l|l|}
\hline Tipo de emoción * Tipo deporte practicado & 12.394 & 6 & .049 \\
\hline Género * Tipo deporte practicado & 2.072 & 2 & .355 \\
\hline Tipo deporte practicado * Nivel competición & .756 & 2 & .685 \\
\hline $\begin{array}{l}\text { Tipo deporte practicado * Huella deportiva Experto-1.855 } \\
\text { novato: años práctica }\end{array}$ & 2 & .396 \\
\hline Tipo deporte practicado * Tipo de resultado & 21.392 & 6 & .002 \\
\hline
\end{tabular}

Tipos de emociones suscitadas en las interacciones motrices de cooperación y de oposición

Las emociones positivas fueron significativamente más elevadas $(M=5.37$; DT $=.451, p<$ $.001, d=7.16$ ) que en el resto de emociones (Figura 5). Nos lleva a un escenario de vivencia claramente positiva, donde las emociones asociadas al malestar casi no cuentan con valoraciones.

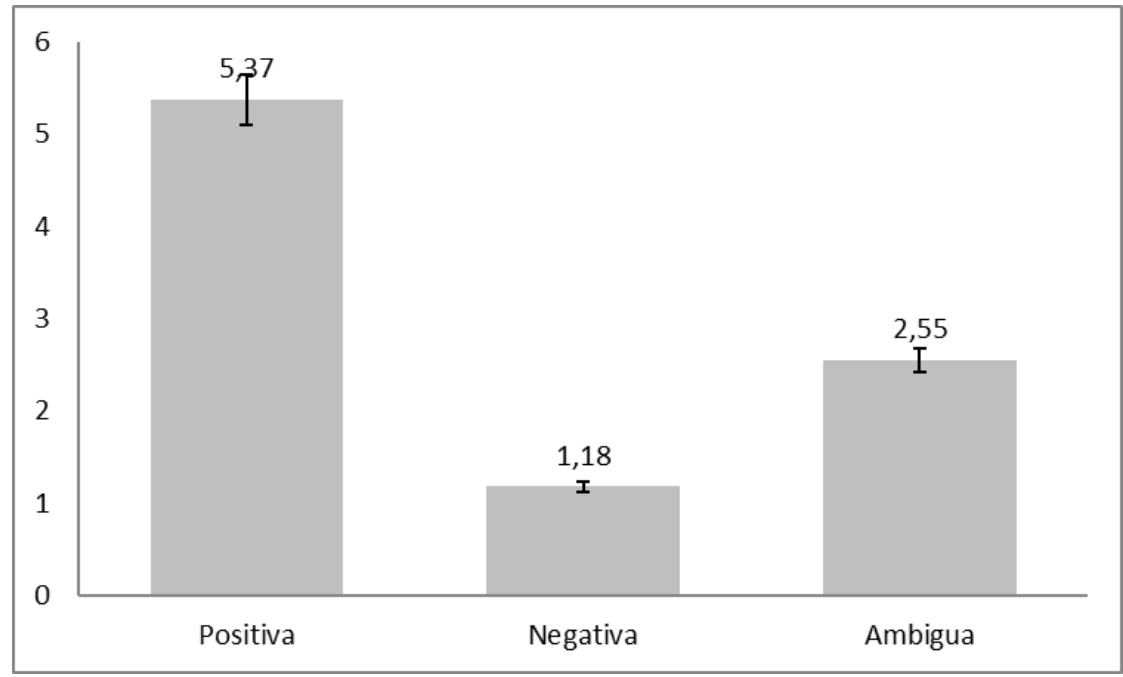

Figura 5. Intensidades de los tres tipos de emociones para la cooperación.

\section{Emociones y competición}

Teniendo en cuenta la presencia o ausencia de la competición en el juego, se observó que las emociones fueron significativamente más intensas $(p<.001)$ al competir y ganar $(M=$ $3.32, \mathrm{DT}=.368, \mathrm{~d}=1.50)$ que al perder $(M=2.90, \mathrm{DT}=.380, \mathrm{~d}=.33)$. No se encontraron diferencias significativas $(p=.865)$ al perder y no competir. Esto nos lleva a que el éxito, conseguir la victoria, fue un factor protagonista en las emociones vividas. A pesar de ser juegos cooperativos, sin la presencia de adversarios que influyan directa y motrizmente, los juegos en los que existió la posibilidad de la victoria, mostraron unas puntuaciones más elevadas. 


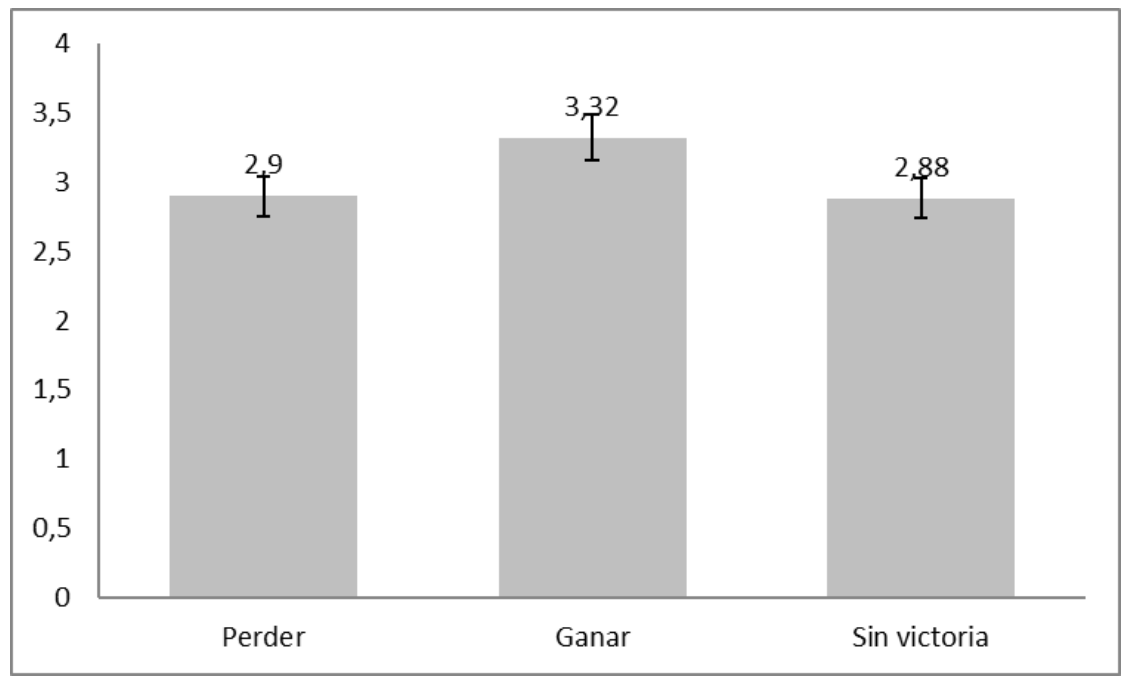

Figura 6. Intensidades emocionales en función del resultado del juego.

\section{Emociones y huella deportiva}

Los resultados de los alumnos que practicaron cooperación y que contaban con historial en deportes cooperativos $(M=1.86)$ registraron valores significativamente inferiores al alumnado con historial en deportes de oposición $(M=4.11$, DT $=.461, p<.001, d=3.77)$. También mostraron valores significativamente inferiores a los del alumnado con historial en deportes psicomotores $(M=3.59, \mathrm{DT}=.447, \mathrm{p}<.001, \mathrm{~d}=1.45)$. Los participantes con huella cooperativa tampoco mostraron diferencias significativas en relación a los que tenían antecedentes en deportes de cooperación-oposición ( $p=.111)$.

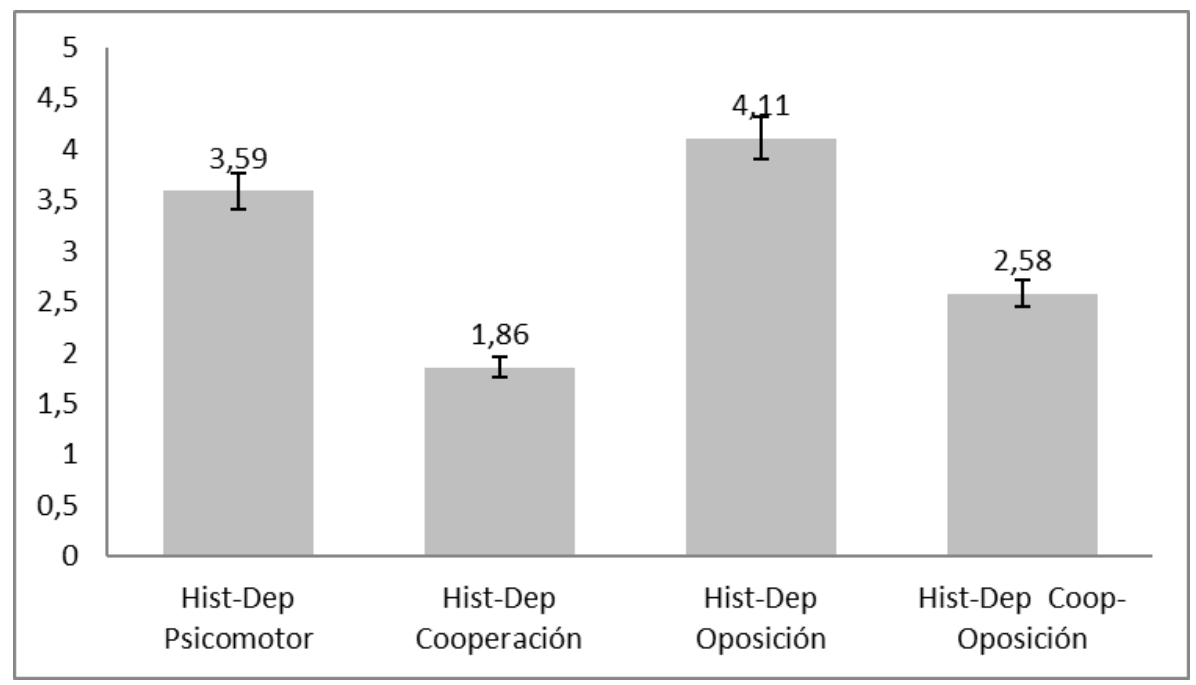

Figura 7. Valores en intensidad emocional para interacciones de oposición y de cooperación según el tipo de historial deportivo.

Dentro de las situaciones cooperativas la vivencia predominante fue positiva, quedando el malestar generado por las emociones negativas muy por debajo. El planteamiento de retos 
cooperativos, con o sin competición, generó una vivencia intensamente positiva en los participantes del estudio. Teniendo en cuenta los datos sobre la experiencia o huella deportiva, los datos no lo remarcan como una variable de peso en la vivencia de los juegos cooperativos. A su vez el género tampoco reportó diferencias significativas respecto de las valoraciones de los participantes.

\section{Discusión}

Los JDT como tipo de interacción motriz desencadenadora de emociones en educación física, han sido analizados en función de variables relacionadas con los propios juegos (dominio de acción y resultado de la interacción motriz) y con variables referidas a los participantes (contar o no con experiencia o huella deportiva, tipo de experiencia y género) dentro de un contexto educativo universitario. Para ello se planteó analizar las situaciones con interacción motriz cooperativa por un lado y de oposición por otro, para observar las reacciones emocionales de los participantes profundizando en el dominio de acción por separado. A su vez, se analizó si la existencia de marcadores finales, como comparador de resultados, podría ser un factor relevante en la vivencia emocional.

En ambas interacciones motrices se suscitaron más emociones positivas que negativas, resultando una experiencia agradable en retos cooperativos y de adversario. Estos mismos resultados fueron encontrados por Lavega et al. (2013), confirmando que las situaciones motrices lúdicas crean un buen clima de aprendizaje para la educación física. Considerar las valoraciones de los tres tipos de emociones, dota al profesor de un indicador pedagógico de gran valor. Unido a la programación de interacciones motrices congruentes con los objetivos pedagógicos, permitiría prever los procesos que pueden desencadenarse al participar en situaciones motrices con estos dos tipos de interacción motriz.

El género no determinó la valoración de las emociones de manera significativa dentro del modelo general, salvo al tener en cuenta los tipos de historial deportivo. Esto implica que las diferencias de la socialización o el aprendizaje emocional a favor de una mayor exteriorización femenina (Fabes y Martín, 1991; Kelly y Hustson-Comeaux, 1999) no se han encontrado en este estudio. Tanto los hombres como las mujeres se expresaron en términos emocionales de forma similar. Este aspecto no concuerda con una mayor riqueza en expresión emocional, mejores conductas prosociales y emociones positivas por parte de las mujeres respecto a los hombres de otros estudios encontrados (Simon y Nath, 2004; Etxebarría et al., 2003).

Parece claro que las diferencias entre hombres y mujeres en contextos deportivos y lúdicos pueden variar respecto a otros contextos analizados por la literatura, lo que iría en la línea de los resultados aportados por Mateu et al. (2014) y Jaqueira et al. (2014). El hecho de que la experiencia deportiva haya motivado diferencias en cuanto a género, permite profundizar en los procesos emocionales de mujeres y hombres. Se puede pensar que, en el ámbito deportivo-federativo, donde se compite duramente, las intensidades emocionales y su regulación pueden verse más influenciadas por la construcción social de las mismas que en el lúdico, por lo que podría ser de gran interés profundizar en este aspecto.

\section{Tipos de emociones suscitadas}

Los JDT proporcionan situaciones motrices que desencadenan experiencias emocionalmente positivas. Con independencia del resto de variables, los resultados van en la dirección apuntada en otras investigaciones (Lavega et al., 2014; Alonso et al., 2013; 
Lavega, et al., 2011, Lavega et al., 2013) que determinaron que la actividad lúdica puede promover conductas prosociales, donde las emociones tienen un papel destacado. Otros estudios indican que, si la vivencia es positiva, las posibilidades de aumentar la motivación y la adherencia hacia la actividad física son más elevadas (Liukkonen et al., 2010). Si entendemos que las interacciones motrices pueden plantearse en contextos como el educativo y generar experiencias positivas, contribuimos a un mejor clima en las aulas, mayor facilidad en la convivencia escolar, así como un aumento en la motivación y participación en las tareas.

Las interacciones durante una tarea motriz permiten intervenir en algunos aspectos de la misma (Bächler y Poblete, 2012) ya que permite acceder a información del otro y ofrece un contexto de conciencia emocional propia y ajena. En el caso de nuestros participantes, como futuros docentes, al ser más conscientes de las emociones positivas de los demás, es más probable que actúen de forma adecuada en otras situaciones cotidianas e integren esta perspectiva en su labor futura (Pena y Extremera, 2012). Además, la naturaleza motriz de las interacciones analizadas pone en acción a la persona desde su globalidad cognitiva, social y emocional, lo que nos acerca a la afectividad (Parlebas, 2001).

Parece coherente que situaciones motrices que desencadenen procesos asociados al pacto, a la superación de un reto o al sacrificio individual por el grupo, hayan disfrutado de una posición relevante en la educación física. No obstante, no existen familias motrices más educativas que otras, sino más o menos congruentes respecto a los objetivos que se planteen (Parlebas, 2001).

Aunque de forma general la experiencia ha sido positiva en ambos tipos de interacción motriz, la posibilidad de cooperar, de sentirse parte de un grupo que busca soluciones a problemas motores, implicaría una mayor percepción de experiencia positiva. Este resultado coincide con la tendencia encontrada por Lavega et al. (2011; 2013) en la que las emociones positivas se asocian a vivencias de tipo cooperativo. Además, investigadores como Castelló y Cano (2011) indican que la existencia de un sentimiento de pertenencia a un grupo ha sido un factor clave en las formas de adaptación humanas, lo que explicaría porque la intensidad emocional vivenciada en la interacción motriz cooperativa ha sido algo más elevada. El sentimiento de protección, ayuda y compartir el reto o problema es un factor a tener en cuenta para futuras investigaciones que tomen la cooperación como forma de interacción.

Los resultados confirman, como en estudios previos, que la vivencia de emociones fue más intensa en competición con marcadores de diversos tipos (Burton-Chellew et al., 2006). Perder o jugar sin marcador no ha sido determinante, entendiendo que jugar sin un final clasificatorio resulta de interés cooperando y oponiéndose. Cabría pensar en otros posibles indicadores de vivencia positiva como la diversión, participación en los juegos, mejora de competencias, experiencias novedosas y compartidas, etc.

En un estudio similar (Lavega et al., 2013), el hecho de ganar en JDT implicó las mayores intensidades emocionales. Se mostró que, en las interacciones cooperativas, ganar no fue determinante para su vivencia emocional. Nuestros datos indican que cuando aparece un adversario, o bien se comparan los resultados, esta tendencia se ve algo modificada. La victoria en el JDT de oposición y en el de cooperación es vivida con intensidad. El enfrentamiento motor que supone un adversario directo conllevaría a una mayor significación en la consecución de la victoria (Lazarus, 2000), así como en la cooperación la existencia de un reto cooperativo, comparando resultados con otros grupos, también genera mayores intensidades emocionales. La tensión generada por la competición es una de las posibles causas de esta diferenciación emocional, independientemente de si 
cooperamos o nos oponemos. La existencia del factor competitivo, al margen del tipo de interacciones motrices de que se trate, provoca que las intensidades emocionales se disparen.

\section{Huella deportiva y género en relación a las vivencias emocionales}

Los resultados indican que, ante la oposición, existe una tendencia a mayores intensidades emocionales cuando los historiales deportivos cuentan con el factor de la oposición. Sin embargo, los historiales cooperativos no muestran diferencia alguna respecto de los historiales con deportes psicomotores. Cuando se practicaron situaciones cooperativas se ha observado que tener antecedentes deportivos individuales y de oposición tiende a desencadenar valores más intensos que cuando el historial es de cooperación o de cooperación-oposición. Los alumnos que se enfrentaron motrizmente mostraron mayores intensidades emocionales cuando su huella deportiva fue sociomotriz. Teniendo en cuenta el género, se confirma esta tendencia de mayor intensidad emocional ante la oposición. En el caso de los chicos con experiencia en deportes sin interacción motriz, su intensidad emocional es inferior al resto de participantes. Esta misma tendencia se observa para las chicas, pero sin la significación estadística del caso anterior. Esta menor vivencia emocional por parte de los alumnos con experiencias individuales puede venir derivada por una mayor regulación y control emocional (Del Pino et al., 2009), donde la concentración previa y atención a las sensaciones propias determinan el rendimiento. De este modo, en situaciones de adversario han podido aplicar estas mismas regulaciones derivando en una vivencia de menor intensidad emocional.

Los alumnos que cooperaron, con experiencia en deportes individuales y de oposición, mostraron mayor intensidad emocional. Aquellos deportistas que han tenido experiencia en prácticas individuales, sin interacción o bien enfrentados a otro deportista, tienden a expresar unas vivencias emocionales mucho más intensas que aquellos con experiencia en deportes de equipo. Por lo tanto, cabe pensar que este tipo de interacción no es una novedad para los expertos, mientras que sí lo es para aquellas personas que no conocen este modo de relacionarse en un deporte.

En las situaciones de oposición para los alumnos con experiencias sociomotrices, es precisamente haber tenido vivencias similares a las tareas planteadas lo que les lleva a una mayor intensidad emocional. Para los alumnos con un historial basado en experiencias deportivas sin interacción motriz, les supone una vivencia de menor intensidad.

Este hecho plantea la necesidad de conocer el tipo de experiencia deportiva previa, ya que supondría una vivencia emocional diferente en cada caso. Si el objetivo es diseñar tareas motrices adecuadas a los objetivos pedagógicos perseguidos, el historial deportivo puede ser un factor relevante en la experiencia educativa. Una adecuada evaluación del tipo de huella deportiva, facilitaría al docente una información de gran valor cuando plantee interacciones motrices cooperativas o de oposición.

\section{Conclusiones}

Teniendo en cuenta lo aportado en el estudio se pueden indicar las siguientes conclusiones:

1. Se han suscitado vivencias emocionales positivas en ambos tipos de interacción motriz, siendo la de tipo cooperativa la de mayor intensidad positiva. 
2. Compitiendo, los ganadores manifiestan una clara tendencia a experimentar elevadas intensidades emocionales en ambos tipos de interacciones motrices.

3. El hecho de ser hombre o mujer no ha sido un factor determinante en las vivencias emocionales.

4. La existencia de huella deportiva, para ambos tipos de interacción motriz, se muestra como una variable a considerar para la evaluación de la vivencia emocional.

Estas conclusiones abren una perspectiva de gran interés para disciplinas que utilicen la interacción motriz como forma de relación psicosocial, como el caso de la enseñanza de la educación física y de los deportes. Con demasiada frecuencia se actúa en educación física de manera excesivamente genérica, habida cuenta de que cualquier tipo de juego desencadena un acusado aumento en las vivencias emocionales. Pero esto, aun siendo constatado por esta investigación, no es suficiente para discriminar la utilización de uno u otro dominio de acción motriz cuando se trata de ajustar la congruencia entre un determinado objetivo pedagógico, en este caso referido a la autorregulación emocional, y el tipo de práctica motriz elegido para lograrlo. Así mismo, la presente investigación estimula el desarrollo de nuevos ámbitos científicos referidos a las vivencias y a la regulación emocional durante las prácticas deportivas en la enseñanza universitaria.

La educación física es un gran laboratorio de interacciones motrices y de vivencias emocionales. Los alumnos ponen en marcha toda su personalidad, afectividad, socialización y motricidad, produciéndose una sucesión de interacciones motrices que deben ser analizadas desde todas sus perspectivas. Los resultados obtenidos muestran que las vivencias emocionales pueden ser un factor de importancia para que tengan éxito dichas interacciones y se facilite el proceso formativo emocional.

\section{Referencias}

Alonso, J.I., Lavega, P. y Gea, G. M. (2013). Incidencia de los juegos de oposición en la vivencia de emociones. En Gázquez et al. (Comps). Investigación en el ámbito escolar. Granada: Ed. GEU.

Bächler, R. y Poblete, O. (2012). Interacción, emoción y cognición: Una aproximación integrada a la comprensión del comportamiento humano. Anales de Psicología, 28(2), 490-504. doi:10.6018/analesps.28.2.147601.

Bisquerra, R. (2003). Educación emocional y competencias básicas para la vida. Revista de Investigación Educativa, 21(1), 7-43.

Burton-Chellewa, M., Ross-Gillespieb, A. \& West, S. (2010). Cooperation in humans: competition between groups and proximate emotions. Evolution and Human Behavior, 31(2), 104-108.

Castelló, A. y Cano, M. (2011). Inteligencia interpersonal: conceptos clave. REIFOP, 14(3), 2335.

Del Pino, J.M., Gómez, E., Moreno, S. y Gálvez, G. (2009). Inteligencias múltiples y deporte. Revista Apunts Educación física y deportes, 95, 5-13.

Etxebarria, I., Apodaca, P., Eceiza, A., Fuentes, Mª J. y Ortiz, Mª J. (2003). Diferencias de género en emociones y en conducta social en la edad escolar. Infancia y Aprendizaje, 26(2), 147-161. 
Etxebeste, J. (2012). À cloche-pied. Les jeux sportifs traditionnels et la socialisation des enfants basques [Hopping on one leg. Traditional sporting games and Basque children's socialization]. Sarrebruck: Editions universitaires europeennes.

Fabes, R. y Martin, C. (1991). Gender and age stereotypes of emotionality. Personality and Social Psychology Bulletin, 17, 532-540.

Gutiérrez, D., González, S., García-López, L. M., \& Mitchell, S. (2011). Differences in decisionmaking development between expert and novice invasion game players. Perceptual and Motor Skills, 112(3), 871-888.

Harris, D. (1981). Personality research: implications for women in sport. In J. Borms, M. Hebbelinck \& A. Venerando (Eds.). The female athlete (pp. 49-57). Basel, NY: Karger.

Jaqueira, A., Lavega, P., Lagardera, F., Araujo, P., y Rodrigues, M. (2014). Educando para la paz jugando: género y emociones en la práctica de juegos cooperativos competitivos. Educatio Siglo XXI, 32(1), 15-32.

Lavega, P., Alonso, J. I., Etxebeste, J., Lagardera, F. \& March, J. (2014). Relationship Between Traditional Games and the Intensity of Emotions Experienced by Participants. Research Quarterly for Exercise and Sport, 85(4), 457-467, DOI: 10.1080/02701367.2014.961048.

Lavega, P., Araujo, P. y Jaqueira, A. (2013). Teaching motor and emotional competencies in university students. Cultura, Ciencia y Deporte, 22(8), 5-15.

Lavega, P., Filella, G., Agulló, M., Soldevila, A., \& March, J. (2011).Understanding emotions through games: Helping trainee teachers to make decisions. Electronic Journal of Research in Educational Psychology, 9, 617-640.

Lavega, P., March, J. y Filella, G. (2013). Juegos deportivos y emociones. Propiedades psicométricas de la escala GES para ser aplicada en la educación física y el deporte. Revista de investigación educativa, 31(1), 151-166.

Lazarus, R. (2000). Estrés y emoción. Manejo e implicaciones en nuestra salud. Bilbao: DDB.

Liukkonen, J., Barkoukis, V., Watt, A., \& Jaakkola, T. (2010). Motivational Climate and Students' Emotional Experiences and Effort in Physical Education. Journal of Educational Research, 103(5), 295-308.

Mateu, M., Romero-Martín, R., Gelpí, P., Rovira, G. y Lavega, P. (2014). La educación emocional a través de las prácticas motrices de expresión. Perspectiva de género. Educatio Siglo XXI, 32(1), 49-70.

Kelly, J. \& Hustson-Comeaux, S. (1999). Gender-emotion stereotypes are context specific. Sex Roles, 40, 107-20.

Parlebas, P. (2001). Juegos, deporte y sociedad. Léxico de praxiología motriz. Barcelona: Paidotribo.

Pena, M. y Extremera, N. (2012). Inteligencia emocional percibida en el profesorado de Primaria y su relación con los niveles de burnout e ilusión por el trabajo (engagement). Revista de Educación, 359, 604-627.

Sáez de Ocáriz, U., Lavega, P., Costes, T. y Serna, J. (2014). ¿Por qué te peleas? Conflictos motores y emociones negativas en la clase de Educación Física: el caso de los juegos de oposición. Educatio Siglo XXI, 32(1), 71-90. 
Simon, R. W. y Nath, L. E. (2004). Gender and emotion in the United States: do men and women differ in self-reports of feelings and expressive behavior? The American Journal of Sociology, 109(5), 1137-1177.

Starkes, J. \& Ericcson, K. (2003). Expert Performance in Sports: Advances in Research on Sport Expertise. Champaign, IL: Human Kinetics.

Velásquez, M. T. y Guillén, N. (2007). Regulación emocional en nadadores en proceso de formación deportiva, categoría infantil. Avances en Psicología Latinoamericana, 25(2), $112-125$.

Wolf, F. M. (1986). Meta-analysis: Quantitative methods for research synthesis. Beverly Hills, CA: Sage. 